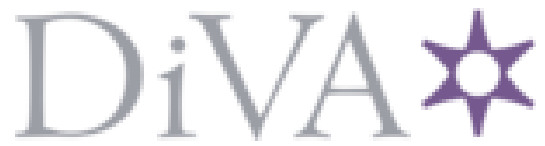

http://www.diva-portal.org

Postprint

This is the accepted version of a paper presented at IEEE International Conference on Robotics and Automation (ICRA).

Citation for the original published paper:

Robert, K., Bekiroglu, Y., Roa, M. (2017)

Grasp Quality Evaluation Done Right: How Assumed Contact Force Bounds Affect Wrench-Based Quality Metrics.

In:

https://doi.org/10.1109/ICRA.2017.7989189

N.B. When citing this work, cite the original published paper.

Permanent link to this version:

http://urn.kb.se/resolve?urn=urn:nbn:se:kth:diva-219543 


\title{
Grasp Quality Evaluation Done Right: How Assumed Contact Force Bounds Affect Wrench-Based Quality Metrics
}

\author{
Robert Krug*, Yasemin Bekiroglu and Máximo A. Roa ${ }^{\ddagger}$
}

\begin{abstract}
Wrench-based quality metrics play an important role in many applications such as grasp planning or grasp success prediction. In this work, we study the following discrepancy which is frequently overlooked in practice: the quality metrics are commonly computed under the assumption of sum-magnitude bounded contact forces, but the corresponding grasps are executed by a fully actuated device where the contact forces are limited independently. By means of experiments carried out in simulation and on real hardware, we show that in this setting the values of these metrics are severely underestimated. This can lead to erroneous conclusions regarding the actual capabilities of the grasps under consideration. Our findings highlight the importance of matching the physical properties of the task and the grasping device with the chosen quality metrics.
\end{abstract}

\section{INTRODUCTION}

Measuring the "goodness" of a grasp is a necessary step in autonomous robotic grasp planning and execution pipelines. To this end, many grasp quality metrics have been proposed over the years (see [1] for a recent survey). Due to their expressiveness and relatively straightforward computation, wrench-based measures are popular in practice. These metrics are derived from the Grasp Wrench Space (GWS) which denotes the set of wrenches (i.e., concatenated force/moment vectors) that can be applied to the object through a given grasp. Computing the GWS and/or related quality metrics entails posing physically meaningful limits on the contact forces that the grasping device can exert. In their seminal work [2], Ferrari and Canny suggested to

i) either assume independent bounds on the maximum magnitude of the contact normal forces,

ii) or assume a bound on their sum-magnitude.

In practice, the contact forces applied by fully actuated robotic grasping systems are generally bounded independently - either by design (e.g., due to actuator limitations) or to avoid damage to hardware and/or the environment. These independent bounds can be enforced using contact force sensors and corresponding control schemes, or by directly limiting hand joint efforts. On the other hand, limiting the contact forces sum-magnitude can be seen as a bound being imposed by a power source shared among all joint actuators of the considered grasping device. Due to computational convenience, this assumption frequently underlies the quality

${ }^{*}$ R. Krug is with the AASS Research Center, Örebro University; 70182 Örebro, Sweden. Email: robert.krug @ oru.se.

${ }^{\ddagger}$ Y. Bekiroglu is with $\mathrm{ABB} A B$, Corporate Research; 72226 Västerås, Sweden. Email: yasemin.bekiroglu@se.abb.com.

${ }^{\diamond} \mathrm{M}$. A. Roa is with the Institute of Robotics and Mechatronics, German Aerospace Center (DLR); 82234 Weßling, Germany. Email: maximo.roa@dlr.de.

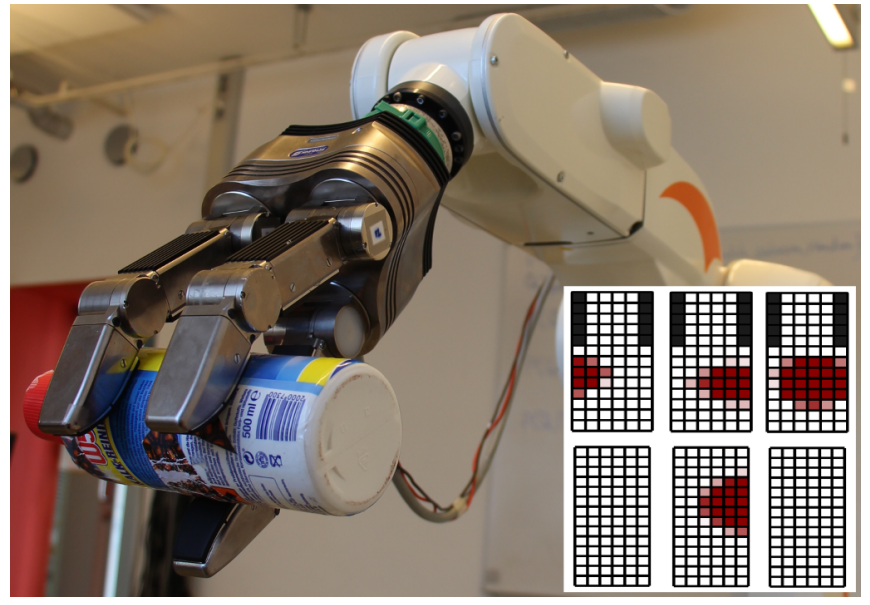

Fig. 1. Platform: The grasps for the experiments in Section IV-B were collected with the depicted three-fingered Schunk Dexterous Hand (SDH) mounted on an industrial KUKA arm. Tactile sensing is enabled by sensor arrays on the two pads of each finger, an exemplary tactile snapshot after grasp acquisition is depicted in the bottom right corner. These arrays comprise $6 \times 14$ taxels on proximal phalanges and $6 \times 13$ taxels on distal phalanges. The hand's $7 \mathrm{DoF}$ are controlled via 7 motors that allow independent joint torque control.

metrics used in grasp planners [3], [4], [5], in synthesis algorithms based on prototype grasps [6], [7], [8] as well as in grasp success prediction systems [9].

However, for the fully actuated hands we consider in this work, it is usually more adequate to consider independent finger force limitations rather than their sum magnitude for grasp quality evaluation [10]. Also, it is important to consider realistic contact force/moment magnitudes opposed to simply assuming them to be unitary as it is common practice. One popular grasp quality metric's sensitivity to pose errors has been investigated in [4]. However, to our knowledge, the impact of the contact bounds assumptions on wrench-based quality metrics has not been studied yet.

The contributions of this work can be summarized as follows. Considering a grasping device with independently enforced contact force limitations we

- experimentally show that the values of wrench-based quality metrics computed according to the commonly made assumption ii) are severely underestimated;

- analyze the practical impact of this underestimation in a grasp success prediction setting by means of a large set of grasps executed by the platform shown in Fig. 1 


\section{BACKGROUND}

The GWS ${ }^{1}$, and therefore the quality metrics derived from it, capture the capability of a grasp to counterbalance external disturbance forces and moments. While often used interchangeably, it is worth noting that equilibrium is necessary but not sufficient for grasp stability and thus grasp success. Indeed equilibrium grasps, and thus their important sub-class of force-closure grasps [11], may be stable or unstable. As pointed out by Bicchi and Kumar [12], any notion of stability (e.g., in the Lyapunov sense) needs to regard the grasp as a dynamic system and describe its behavior when perturbed from equilibrium. However, analyzing dynamic stability is difficult in practice as it requires to include local contact curvature, mechanical compliance, hand control laws (a compliant controller which allows hand-relative object movement results in a change of GWS) as well as the magnitude and arrangement of the applied contact forces [13], [14]. Nevertheless, our previous work [15] showed that realistic contact force modeling, involving tactile feedback and joint effort limits, allows wrench-based reasoning to predict grasp success surprisingly well and on-par with recent approaches based on supervised learning [9], [16], [17].

As discussed in more detail below, the reason why wrenchbased quality metrics are often computed according to assumption ii) in Section II lies in the fact that for many applications constructing the GWS using the usually more realistic assumption i) is only feasible for a small number of contacts or crude approximations of contact friction [3]. In this work, we study in depth the consequences of using metrics computed according to assumption ii) to assess the quality of grasps to be executed by a fully actuated device with independently limited contact forces. Note that assumption i) is meaningless for a fully underactuated hand (only a single actuator driving all DoF), like a parallel jaw gripper. Metrics for such devices should indeed be computed with a sum-magnitude bound assumption. For a detailed study of the influence of underactuation on the grasp quality measure computation we refer the reader to [18].

\section{A. Grasp Contact Modeling}

A grasp is described as $i=1, \ldots, n$ soft contacts that form contact areas $\mathcal{C}_{i} \subset \mathbb{R}^{3}$ on the target object surface. Each pressure-weighted contact center $\boldsymbol{p}_{i} \in \mathbb{R}^{3}$ is associated with an inward-pointing surface unit normal $\boldsymbol{n}_{i} \in \mathbb{R}^{3}$. Considering a local contact frame with origin at $\boldsymbol{p}_{i}$ and $z$-axis pointing along $\boldsymbol{n}_{i}$, we denote a generalized contact friction force $\boldsymbol{f}=\left[f_{x}, f_{y}, f_{z}, \tau_{z}\right]^{T}$. Here, $f_{x, y}$ and $f_{z}$ respectively indicate tangential and normal contact force components, while $\tau_{z}$ stands for the frictional moment about the contact normal. As proposed by Howe and Cutkosky [19], we formulate an ellipsoid limiting the set of frictional forces/moments that can be transmitted by a deformable finger contact onto

\footnotetext{
${ }^{1}$ Since the identity- and inverse elements of addition axioms do not hold in general, the Grasp Wrench Space is a set but not a vector space. Nevertheless, we adhere to the naming convention made in the literature.
}
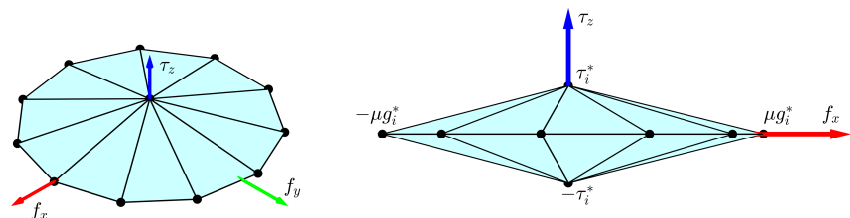

Fig. 2. Discretized limit surface: Depicted are two views of the friction ellipsoid which, for the experiments reported in Section IV] we approximate by a polytope with 14 vertices. The vertices correspond to the apices and equidistant samples drawn from the circular base of the ellipsoid in 1 .

a flat surface with friction coefficient $\mu \in \mathbb{R}_{+}$

$$
\mathcal{F}_{i}=\left\{\boldsymbol{f} \mid f_{x}^{2}+f_{y}^{2}+\left(\mu f_{z} \tau_{z} / \tau_{i}^{*}\right)^{2} \leq\left(\mu f_{z}\right)^{2}, f_{z}=g_{i}^{*}\right\}
$$

Formulation (1) depends on the maximum normal force $g_{i}^{*} \in \mathbb{R}_{+}$and the maximum frictional moment $\tau_{i}^{*} \in \mathbb{R}_{+}$that the soft contact can apply under this normal force. The apices $\pm \tau_{i}^{*}$ of the friction ellipsoid correspond to pure torsional load. Once the grasp is loaded, a deformable fingertip will allow some object motion. However, in response the pressure distribution will change and thus limit that motion. This factuality can be modeled by appropriately augmenting the set of exertable forces and moments. Here, we build upon the approach in [20] and apply the generalized frictional forces in (1) over the whole contact surface area. Correspondingly, we express the set of wrenches a contact can exert as

$$
\mathcal{W}_{i}=\left\{\boldsymbol{w} \in \mathbb{R}^{6} \mid \boldsymbol{w}=\boldsymbol{G}(\boldsymbol{c}) \boldsymbol{f}, \boldsymbol{c} \in \mathcal{C}_{i}, \boldsymbol{f} \in \mathcal{F}_{i}\right\},
$$

where the matrix $\boldsymbol{G}(\boldsymbol{c}) \in \mathbb{R}^{6 \times 4}$ maps generalized forces to wrenches expressed in an object coordinate frame [21]. Let us discretize $\mathcal{W}_{i}$ in 2 by a total of $l_{i}$ samples and collect them in the column-matrix

$$
\boldsymbol{W}_{i}=\left[\boldsymbol{w}_{i, 1}, \ldots, \boldsymbol{w}_{i, l_{i}}\right]
$$

The discretization in (3) corresponds to drawing samples from the friction ellipsoids (see Fig. 2) associated with each taxel center $\boldsymbol{c}_{i}\left(x_{i}, y_{i}\right)$ having non-zero tactile readings.

As mentioned before, to formulate the set of wrenches that a grasp can apply, it is necessary to assume bounds on the contact normal forces. As shown in [2], posing independent (uniform) bounds can be expressed by using a $L_{\infty}$ metric in the norm definition of the vector formed by the $n$ individual contact normal force components. Similarly, the contact normal force sum magnitude can be limited by assuming a $L_{1}$ metric. Correspondingly, the discrete GWS ca be found by either forming the convex hull over the Minkowski sum of the $n$ contact wrench sets in (3)

$$
\mathrm{GWS}_{L_{\infty}}=\operatorname{conv}\left(\bigoplus_{i=1}^{n}\left\{\boldsymbol{w}_{i, 1}, \ldots, \boldsymbol{w}_{i, l_{i}}\right\}\right)
$$

or via the convex hull over the union of contact wrenches

$$
\operatorname{GWS}_{L_{1}}=\operatorname{conv}\left(\bigcup_{i=1}^{n}\left\{\boldsymbol{w}_{i, 1}, \ldots, \boldsymbol{w}_{i, l_{i}}\right\}\right) \text {. }
$$

Note, that $\mathrm{GWS}_{L_{\infty}}$ is a superset of $\mathrm{GWS}_{L_{1}}$ [2]. 


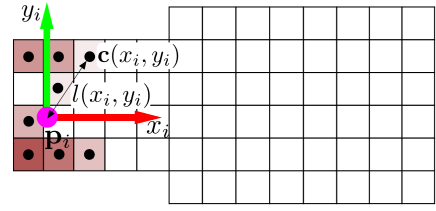

Fig. 3. Computing maximum moment $\tau_{i}^{*}$ : In the case of pure torsional load, the frictional force acting at a taxel center $c\left(x_{i}, y_{i}\right)$ is at most $\frac{\mu g_{i}^{*}}{a} \hat{g}_{i}\left(x_{i}, y_{i}\right)$. Multiplying with the distance to pressure center $\boldsymbol{p}_{i}$ yields the corresponding moment contributions which are summed up to give the overall moment $\tau^{*}$ in 7 .

\section{B. Contact Parametrization}

For the real-world grasp success prediction experiments in Section IV-B, we exploit given hand joint torque limits and tactile feedback after grasp acquisition to obtain realistic estimates of the maximum contact forces/moments in (1). As discussed in [15], this entails approximating the contact surfaces $\mathcal{C}_{i}$ by projecting the corresponding taxel grids from the curved finger pads onto planes tangential to the pressureweighted centers $\boldsymbol{p}_{i}$. Then, the exertable contact forces and moments (which differ between contacts in general) are obtained and used to compute the contact wrench set in (2).

To this end, we denote the hand joint configuration as $\boldsymbol{q} \in \mathbb{R}_{+}^{k}$ and the vector containing the maximum joint torques as $\bar{\tau} \in \mathbb{R}_{+}^{k}$. Assuming negligible external loads such as gravity or inertial forces, the joint torques can be expressed as the product of the total contact force and the transpose of the hand jacobian $\boldsymbol{J}_{i}(\boldsymbol{q}) \in \mathbb{R}^{3 \times k}$ formed with respect to $\boldsymbol{p}_{i}$. This relation allows to obtain the maximum normal force at the $i^{\text {th }}$ contact by solving the Linear Program (LP)

$$
\begin{gathered}
g_{i}^{*} \in \underset{g_{i} \in \mathbb{R}_{+}}{\arg \max } g_{i}, \\
\text { subject to }-\overline{\boldsymbol{\tau}} \leq \boldsymbol{J}_{i}(\boldsymbol{q})^{T} \boldsymbol{n}_{i} g_{i} \leq \overline{\boldsymbol{\tau}} .
\end{gathered}
$$

According to [19], the maximum moment $\tau_{i}^{*}$ can be estimated by integrating the frictional moment contributions made by the local tangential forces acting at individual taxel centers $\boldsymbol{c}_{i}\left(x_{i}, y_{i}\right)$. To this end, let $a=\sum_{\mathcal{C}_{i}} \hat{g}_{i}\left(x_{i}, y_{i}\right)$ be the sum of raw tactile readings over contact area $\mathcal{C}_{i}$ and $l\left(x_{i}, y_{i}\right)$ be the euclidean distance between pressure center and a taxel center. This allows to write the searched for moment as

$$
\tau_{i}^{*}=\frac{\mu g_{i}^{*}}{a} \sum_{\mathcal{C}_{i}} \hat{g}_{i}\left(x_{i}, y_{i}\right) l\left(x_{i}, y_{i}\right)
$$

The corresponding quantities are illustrated in Fig. 3 Equation (7) implies that the measured readings $\hat{g}_{i}\left(x_{i}, y_{i}\right)$ correspond to the maximal possible normal force magnitude $g_{i}^{*}$, which is not the necessarily the case. Nevertheless, $\tau_{i}^{*}$ is conservatively approximated by (7) because the contact surface resulting from $g_{i}^{*}$ will be larger or equal than $\mathcal{C}_{i}$.

\section{WRENCH-BASED GRASP QuAlity Metrics}

At bottom, grasp success depends on whether the hand is able to exert wrenches suitable to counterbalance external disturbances. Therefore, as pointed out by Borst et al. [22], a good quality measure is a scalar describing how well the grasp can resist external disturbance wrenches, $i . e$, how
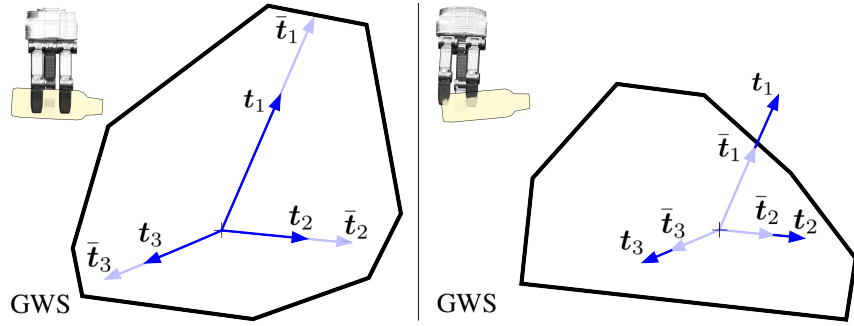

Fig. 4. Task wrench set scaling: To facilitate understanding, the taskrelated quality criterion proposed in [24] is illustrated in a hypothetica $2 \mathrm{~d}$ wrench space. The metric $q^{*}$ is the largest scaling factor of the task wrenches $\boldsymbol{t}_{j}$, such that all scaled wrenches $\overline{\boldsymbol{t}}_{j}$ lie inside the GWS, i.e., $\overline{\boldsymbol{t}}_{j}=q^{*} \boldsymbol{t}_{j}, j=1, \ldots, 3$. (Left) The grasp corresponding to the depicted GWS is able to exert all task wrenches $\left(q^{*} \geq[1)\right.$. (Right) As the grasp can not counterbalance all disturbances, not all task wrenches are contained in the GWS $\left(q^{*}<1\right)$.

suitable it is to accomplish the task at hand. To investigate the influence of the chosen grasp contact force bounds, we consider the three wrench-based metrics outlined below. They are computed using the maximum normal contact forces/moments according to (6) and (7) respectively.

\section{A. Largest-Minimum Resisted Wrench}

To qualify the subset of force-closure grasps, if nothing about the task is known, a popular metric was proposed by Kirkpatrick et al. [23]. It is defined as the magnitude of the largest perturbation wrench that can be resisted in any direction or formally

$$
\epsilon=\min _{\boldsymbol{w} \in \delta \mathrm{GWS}}\|\boldsymbol{w}\|_{2},
$$

where $\delta \mathrm{GWS}$ denotes the boundary of the GWS according to either (4) or (5]. Note that $\epsilon_{L_{\infty}} \geq \epsilon_{L_{1}}$ [2]. Geometrically, this criterion represents the radius of the largest origin-centered ball in the wrench space.

\section{B. Grasp Wrench Space Volume}

The metric $V_{\mathrm{GWS}}$ is given by the volume of the GWS according to either (4) or (5). This measure is independent of the reference system used to compute moments, but does not indicate potential weaknesses of the grasp in certain wrench directions [1].

\section{Task Wrench Set Scaling Factor}

It has been recognized that a meaningful task description should involve the wrenches which a grasp needs to exert in order to counter the disturbance forces/moments occurring during task execution [25], [22], [6], [26]. In this line of thought, Haschke et al. [24] suggested to evaluate the containment of a set of task wrenches in the GWS. This entails to describe the task as a set formed by the mirror image of expected disturbance wrenches that need to be resisted

$$
\mathcal{T}=\left\{\boldsymbol{t}_{j} \in \mathbb{R}^{6} \mid j=1, \ldots, m\right\} .
$$

While it might be difficult to formulate the task wrench set in (8) for general tasks, one option is to model it by means of demonstrations [27]. The corresponding quality metric constitutes the maximum task wrench set scaling factor $q^{*}$ 


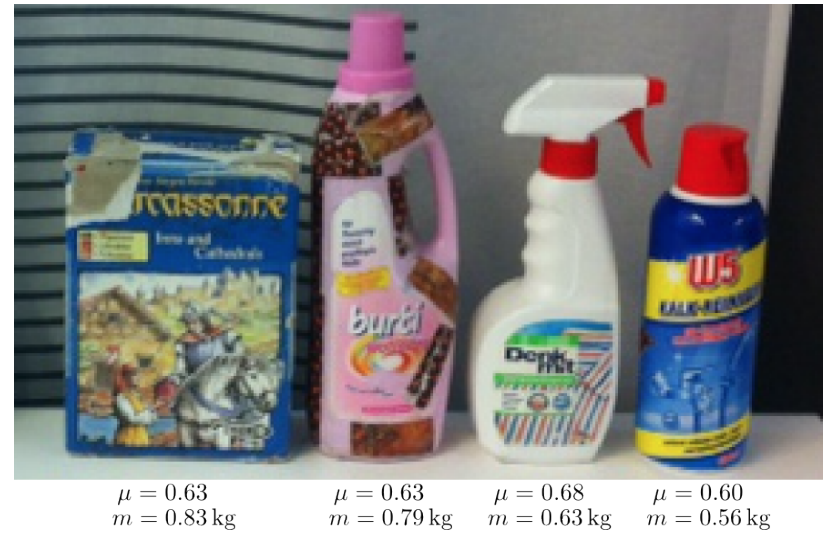

Fig. 5. Test objects: The four objects used in the evaluation and their respective masses and friction coefficients. Given in brackets is the respective number of real-world grasps used for the success prediction experiments in Section IV-B From left: Box (134 grasps), Oval Bottle (170 grasps), Spray Bottle (142 grasps) and Cylindrical Bottle (138 grasps).

such that the GWS according to either (4) or (5) contains the task set in (8) as illustrated in Fig. 4. Therefore, $q^{*} \geq 1$ constitutes the decision boundary at which we expect a grasp to be successful, since it should be able to counterbalance all expected disturbance wrenches. Opposed to the similar $Q$ distance criterion by Zhu and Wang [25], this criterion does not require the task wrench set to contain the origin. Also, it does not necessitate the force-closure property and is defined for non-prehensile grasps. Computation of $q^{*}$ in [24] requires, for each wrench in (8), the solution of a determinant maximization problem with linear matrix inequality constraints. In [15] we proposed an efficient computation of $q^{*}$ for independently bounded grasp contact forces as the solution of a Linear Program (LP) for which highly efficient solvers exist. The formulation expresses each of the scaled task wrenches in (8) as convex combinations of the discrete grasp contact wrenches in (3) summed over all contacts

$$
q_{L_{\infty}}^{*} \in \underset{q_{L_{\infty}} \in \mathbb{R}_{+}, \boldsymbol{\lambda}_{i, j} \in \mathbb{R}_{+}^{l_{i}}}{\arg \max } q_{L_{\infty},}
$$

subject to

$$
\begin{aligned}
\sum_{i=1}^{n} \boldsymbol{W}_{i} \boldsymbol{\lambda}_{i, j} & =q_{L_{\infty}} \boldsymbol{t}_{j}, \quad j=1, \ldots, m, \\
\left\|\boldsymbol{\lambda}_{i, j}\right\|_{1} & =1, \quad i=1, \ldots, n, j=1, \ldots, m .
\end{aligned}
$$

To obtain the analogous metric under the assumption of a sum-magnitude bound on the grasp contact forces, let us collect the $n$ grasp contact wrench matrices in (3) in the column matrix $\boldsymbol{W}=\left[\begin{array}{lll}\boldsymbol{W}_{1} & \cdots & \boldsymbol{W}_{n}\end{array}\right]$. This allows to formulate the LP

$$
q_{L_{1}}^{*} \in \underset{q_{L_{1}} \in \mathbb{R}_{+}, \quad \boldsymbol{\lambda}_{j} \in \mathbb{R}_{+}^{\sum_{i=1}^{n} l_{i}}}{\arg \max } q_{L_{1}},
$$

subject to

$$
\begin{array}{rlrl}
\boldsymbol{W} \boldsymbol{\lambda}_{j} & =q_{L_{1}} \boldsymbol{t}_{j}, & j=1, \ldots, m, \\
\left\|\boldsymbol{\lambda}_{j}\right\|_{1} & =1, & j & =1, \ldots, m .
\end{array}
$$

Note that, opposed to the $\epsilon$ and $V_{\mathrm{GWS}}$ metrics discussed before, neither the formulation in 9 nor in 10 requires

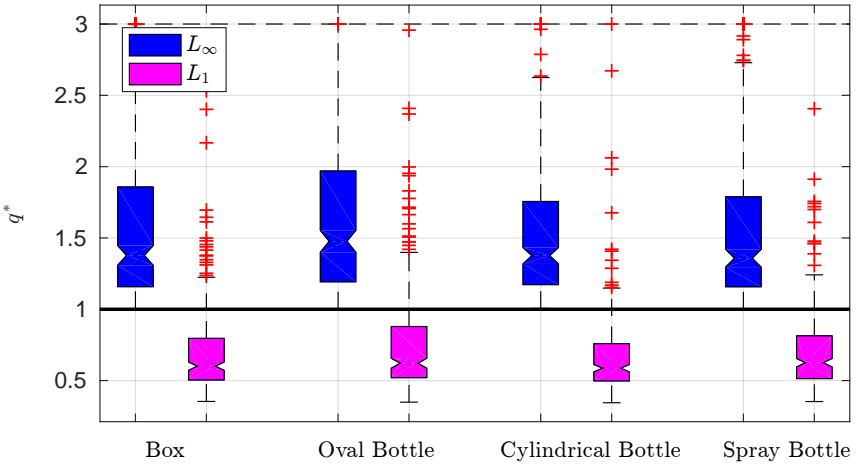

Fig. 6. Simulation results for $q^{*}$ : The obtained task wrench set scaling factors with contact normal forces respectively assumed to be sum-magnitude or independently bounded (outliers are clipped at $q^{*}=3$ ).

TABLE I

SimUlation RESUlTS OF THE EXPERIMENTS In SECTION IV-A

\begin{tabular}{|c|c|c|c|c|c|}
\hline & $q^{*}$ & $V_{\text {GWS }}\left[\times 10^{-3}\right]$ & $\epsilon\left[\times 10^{-3}\right]$ & $t_{q^{*}}[\mathrm{~ms}]$ & $t_{\mathrm{GWS}}[\mathrm{s}]$ \\
\hline \hline$L_{\infty}$ & $1.64 \pm 0.8$ & $10.59 \pm 10.0$ & $13.97 \pm 9.5$ & $0.33 \pm 0.1$ & $9.04 \pm 1.1$ \\
\hline$L_{1}$ & $0.72 \pm 0.4$ & $0.11 \pm 0.1$ & $6.04 \pm 4.0$ & $0.38 \pm 0.1$ & $0.06 \pm 0.0$ \\
\hline
\end{tabular}

the actual construction of the GWS.

\section{EXPERIMENTAL EVALUATION}

The concepts described in Sections II and III were implemented in Matlab and the following results were generated on a standard PC with 6 GB RAM and an Intel i7 - 2600 CPU. An off-the-shelf solven was used to solve the LP's in (6), (9) and (10). For evaluation purposes, we used the 4 test objects depicted in Fig. 5 and their corresponding discretized mesh representations.

\section{A. Evaluation in Simulation}

For a first set of experiments, we assumed unit contact normal forces and generated 250 hard-finger grasps (i.e., the moment about the contact normal $\tau_{i}^{*}=0$ in (1) and the contact surfaces $\mathcal{C}_{i}$ in (2) only contained a single point) with 3 contact points on the discretized model of each test object. Every test grasp was associated with a separate, randomly generated task wrench with magnitude between 0 and 1 . The grasps where generated randomly, but grasps with a quality criterion $q_{L_{\infty}}^{*} \leq 1$ according to 9 were discarded i.e., every grasp was able to counterbalance its associated disturbance wrench under the assumption of independent contact force bounds. Out of the 1000 grasps, 419 happened to be force-closure $(41.9 \%)$. While respectively assuming independent as well as sum-magnitude bounded contact normal forces, we constructed the GWS and the quality measures described in Sections III-B and III-C for all grasps. The $\epsilon$ measures according to Section III-A were computed only for the subset of force-closure grasps.

The mean and 1-STD values of the results are summarized in Tab. I Figure 6 separately depicts the obtained task wrench set scaling measures according to 9 and 10

http://www.gurobi.com/ 


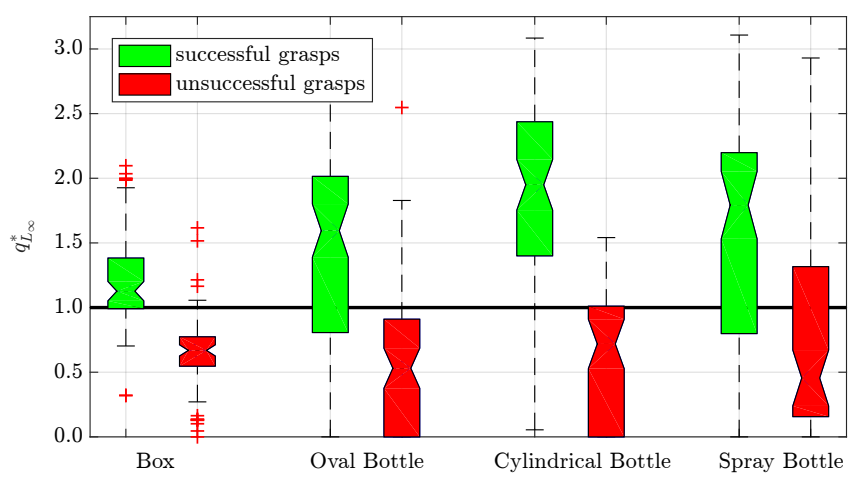

(a)

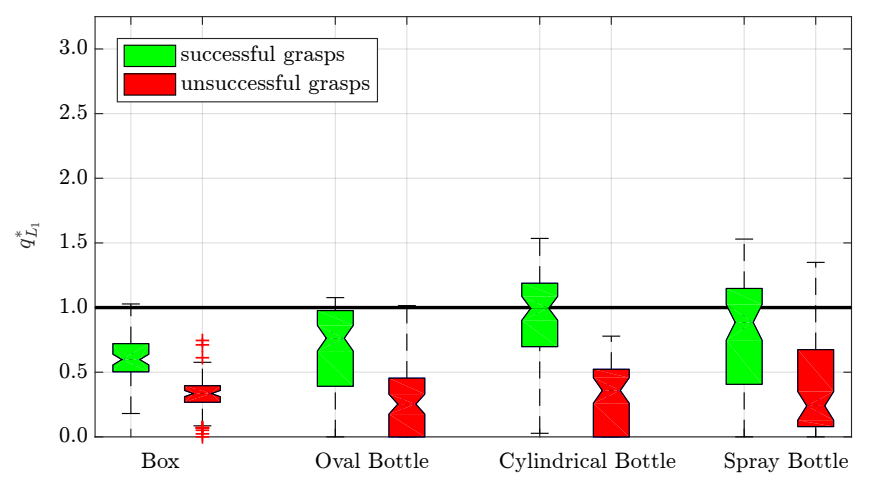

(b)

Fig. 7. Real-world results for $q^{*}$ : (a) the distribution of the metric computed under the assumption of independently bounded contact forces according to 9; (b) results for the assumption of sum magnitude bounded contact forces according to 10.

respectively. Both the $\epsilon_{L_{\infty}}$ and $q_{L_{\infty}}^{*}$ metrics are roughly $130 \%$ larger than their counterparts computed under the sum-magnitude bound assumption, while the $V_{\mathrm{GWS}}$ metrics differ by two orders of magnitude. For the common case of grasping devices with independently limited contact forces, the obtained results highlight the fact that metrics computed using a sum-magnitude bound severely underestimate the actual quality values. Furthermore, by construction, all test grasps were able to counterbalance the given task wrench (i.e., $q_{L_{\infty}}^{*} \geq 1$ ). Nevertheless, according to the $q_{L_{1}}^{*}$ metrics, $86.7 \%$ of the grasps are incorrectly predicted as failures (i.e., $q_{L_{1}}^{*}<1$ ). The mean computation times for constructing the GWS are two orders of magnitude higher when considering independent contact force bounds $(9.04 \mathrm{~s}$ vs. $60 \mathrm{~ms}$ ). Interestingly however, the mean computation times for $q_{L_{\infty}}^{*}$ and $q_{L_{1}}^{*}$ are comparable at around $0.35 \mathrm{~ms}$.

\section{B. Grasp Success Prediction in Practice}

We conducted experiments using the hand shown in Fig. 1 to test the $q_{L_{\infty}}^{*}$ and $q_{L_{1}}^{*}$ metrics regarding their ability to predict ground-truth grasp success rates measured from realworld experiments. We collected a data set comprising 584 precision grasps on the test objects shown in Fig. 5. Half of these grasps remained stable while lifting the grasped object, whereas the other half failed to prevent object slippage and/or rotation. To register an object to the robot reference frame, we tracked the pose of a corresponding textured CAD model in a monocular video stream using the system in [28]. Tracking object movement for the complete duration of the grasp and using previous frames is more robust than performing pose estimation at the end of the movement only. We use tactile feedback obtained after grasp acquisition to parametrize the contact model as discussed in Section II-B. The lifting task was modeled with a wrench set in 8 corresponding to the gravity force distributed in a radius of $6.7 \mathrm{~mm}$ around the object's center of mass to account for perception uncertainties (see [15] for a sensitivity analysis regarding the task parametrization). For a more detailed description of the experimental setting and the used parameters we refer the reader to [15].

We respectively computed the task wrench set scaling factors according to (9) and (10) for all grasps. Figure 7 illustrates the distribution of the obtained metrics. Consistent with the simulation results reported in the previous section, the obtained factors $q_{L_{\infty}}^{*}$ are approximately twice as large as the corresponding $q_{L_{1}}^{*}$ values. Therefore, assuming sum magnitude bound contact forces results in an underestimation of the actual grasp quality as highlighted by the classification accuracy depicted in Fig. 8. While the overall accuracies are relatively close, it is evident that using the $q_{L_{1}}^{*}$ metric for classification results in disproportionally low true positive rates (as low as $4.5 \%$ in case of the Box object). The key insight is that, due to the fact that $\mathrm{GWS}_{L_{1}} \subseteq \mathrm{GWS}_{L_{\infty}}$, using the sum-magnitude bound assumption will result in overly conservative success prediction. In our experiments, the $q_{L_{\infty}}^{*}$ metric yielded $25 \%$ false positives over all trials opposed to only $2 \%$ for the $q_{L_{1}}^{*}$ metric. Nevertheless, by simply shifting the decision boundary, success prediction with the $q_{L_{\infty}}^{*}$ metric can be made as conservative as desired (see [15]).

Some misclassifications remain even under the more realistic $L_{\infty}$ norm assumption on the contact force bounds in agreement with our initial observations in [15]. We attribute this to inaccuracies in the contact modeling and the fact that wrench-based reasoning is blind to mechanical compliance, as well as to unmodeled controller dynamics. A compliant controller and/or object allows for hand-relative object motion when the grasp is loaded during task execution. This can change the set of exertable contact wrenches in (2), as well as the task wrench set in 8 ( $)$ e.g., due to a hand-relative change of the weight vector coordinates) and the hand-configuration dependent maximum contact normal force and moment in (6) and (7) respectively. Furthermore, during the experiments we could observe that hand-controller induced fingertip motion was frequently responsible for unsuccessful grasps caused by slippage if the contacts occurred near the fingertip borders.

\section{CONCLUSIONS}

In this paper we study how the assumptions made on grasp contact force bounds affect three well-known wrenchbased grasp quality metrics. We investigate the two cases of sum-magnitude bounded contact forces (according to a $L_{1}$ norm) or independently limited forces (according to a $L_{\infty}$ norm). Our findings are based on simulations and real-world 


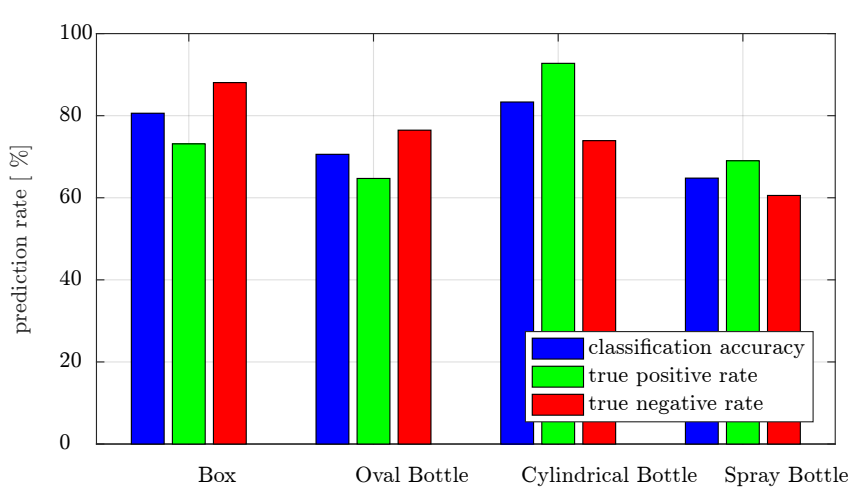

(a)

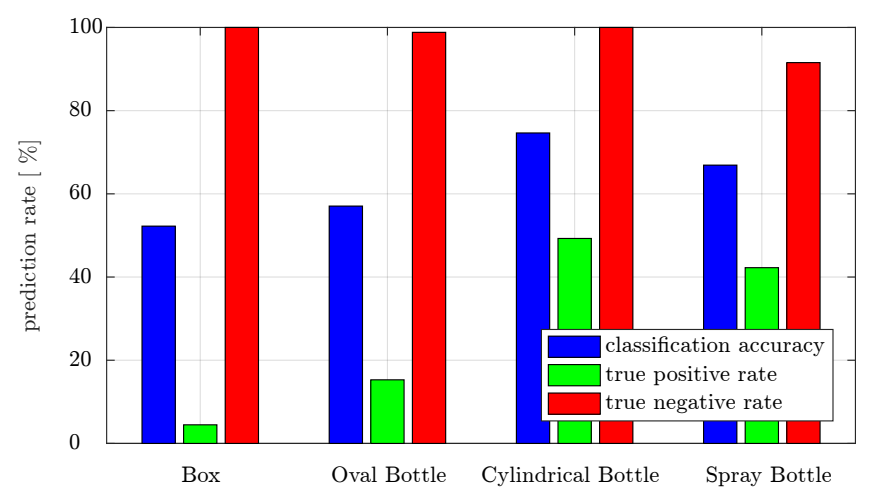

(b)

Fig. 8. Real-world grasp prediction results: (a) The bar graph illustrates the prediction rates when classifying with a decision boundary of $q_{L_{\infty}}^{*} \geq 1$, overall accuracy is $75 \%$ (cf. [15]). (b) prediction rates when using $q_{L_{1}}^{*} \geq 1$ as decision boundary, overall accuracy is $63 \%$.

experiments in a grasp success prediction scenario. We show that, for precision grasps collected with a three-fingered fully actuated hand, the sum-magnitude bound assumption yields metrics that are between a factor of two and two order of magnitudes smaller than their counterparts computed under independent force limits. Also, the $L_{1}$ norm metric fails on predicting grasp success even for grasps that are constructed as force-closure grasps.

Our work therefore highlights the importance of matching the physical properties of the grasping device with the underlying assumptions made during grasp quality assessment. In practice, there is often a mismatch since the contact forces exerted by fully actuated grasping devices are limited independently. However, for applications where computational efficiency is key, wrench-based metrics are often computed under a sum-magnitude force bound assumption. This is to avoid the computation of the full Grasp Wrench Space in $\mathcal{H}$-representation which is expensive when assuming independent force limits. Instead, we suggest to compute the corresponding metrics incrementally [10], via ray-shooting, or to employ metrics which altogether avoid the GWS computation as discussed in Section III-C.

\section{ACKNOWLEDGMENTS}

This work was supported in part by the EU H2020 projects ILIAD (732737) and SARAFun (644938).

\section{REFERENCES}

[1] M. A. Roa and R. Suárez, "Grasp quality measures: review and performance," AURO, vol. 38, no. 1, pp. 65-88, 2015.

[2] C. Ferrari and J. Canny, "Planning optimal grasps," in Proc. IEEE ICRA, 1992, pp. 2290-2295.

[3] A. T. Miller and P. K. Allen, "Graspit! a versatile simulator for robotic grasping," RAM, vol. 11, no. 4, pp. 110-122, 2004.

[4] J. Weisz and P. K. Allen, "Pose error robust grasping from contact wrench space metrics," in Proc. IEEE ICRA, 2012, pp. 557-562.

[5] T. Stoyanov, N. Vaskevicius, C. A. Müller, et al., "No more heavy lifting: Robotic solutions to the container unloading problem," $R A M$, vol. 23, no. 4, pp. 94-106, 2016.

[6] N. S. Pollard, "Closure and quality equivalence for efficient synthesis of grasps from examples," IJRR, vol. 23, no. 6, pp. 595-613, 2004.

[7] M. A. Roa and R. Suárez, "Computation of independent contact regions for grasping 3-d objects," T-RO, vol. 25, no. 4, pp. 839-850, 2009.
[8] R. Krug, D. Dimitrov, K. Charusta, and B. Iliev, "On the efficient computation of independent contact regions for force closure grasps," in Proc. IEEE/RSJ IROS, 2010, pp. 586-591.

[9] Y. Bekiroglu, J. Laaksonen, J. A. Jørgensen, V. Kyrki, and D. Kragic, "Assessing grasp stability based on learning and haptic data," T-RO, vol. 27, no. 3, pp. 616-629, 2011.

[10] C. Borst, M. Fischer, and G. Hirzinger, "A fast and robust grasp planner for arbitrary 3d objects," in Proc. IEEE ICRA, vol. 3, 1999, pp. 1890-1896.

[11] A. Bicchi, "On the closure properties of robotic grasping," IJRR, vol. 14, no. 4, pp. 319-334, 1995.

[12] A. Bicchi and V. Kumar, "Robotic grasping and contact: A review," in Proc. IEEE ICRA, 2000, pp. 348-353.

[13] C.-H. Xiong, Y.-F. Li, H. Ding, and Y.-L. Xiong, "On the dynamic stability of grasping," IJRR, vol. 18, no. 9, pp. 951-958, 1999.

[14] A. Shapiro, E. Rimon, and A. Ohev-Zion, "On the mechanics of natural compliance in frictional contacts and its effect on grasp stiffness and stability," IJRR, vol. 32, no. 4, pp. 425-445, 2013.

[15] R. Krug, A. J. Lilienthal, D. Kragic, and Y. Bekiroglu, "Analytic grasp success prediction with tactile feedback," in Proc. IEEE ICRA, 2016, pp. $165-171$.

[16] H. Dang and P. K. Allen, "Stable grasping under pose uncertainty using tactile feedback," AURO, vol. 36, no. 4, pp. 309-330, 2014.

[17] A. K. Goins, R. Carpenter, W.-K. Wong, and R. Balasubramanian, "Implementation of a gaussian process-based machine learning grasp predictor," AURO, pp. 1-13, 2015.

[18] M. Pozzi, A. M. Sundaram, M. Malvezzi, D. Pratichizzo, and M. A. Roa, "Grasp quality evaluation in underactuated robotic hands," in Proc. IEEE/RSJ IROS, 2016, pp. 1946-1953.

[19] R. D. Howe and M. R. Cutkosky, "Practical force-motion models for sliding manipulation," IJRR, vol. 15, no. 6, pp. 557-572, 1996.

[20] K. Charusta, R. Krug, D. Dimitrov, and B. Iliev, "Independent contact regions based on a patch contact model," in Proc. IEEE ICRA, 2012, pp. 4162-4169.

[21] R. M. Murray, Z. Li, and S. S. Sastry, A mathematical introduction to robotic manipulation. CRC press, 1994.

[22] C. Borst, M. Fischer, and G. Hirzinger, "Grasp planning: How to choose a suitable task wrench space," in Proc. IEEE ICRA, vol. 1, 2004, pp. 319-325.

[23] D. Kirkpatrick, B. Mishra, and C.-K. Yap, "Quantitative Steinitz's theorems with applications to multifingered grasping," Discrete \& Computational Geometry, vol. 7, no. 1, pp. 295-318, 1992.

[24] R. Haschke, J. J. Steil, I. Steuwer, and H. Ritter, "Task-oriented quality measures for dextrous grasping," in Proc. IEEE CIRA, 2005, pp. 689694.

[25] X. Zhu and J. Wang, "Synthesis of force-closure grasps on 3-d objects based on the $Q$ distance," T-RO, vol. 19, no. 4, pp. 669-679, 2003.

[26] H. Kruger, E. Rimon, and A. F. van der Stappen, "Local force closure," in Proc. IEEE ICRA, 2012, pp. 4176-4182.

[27] Y. Lin and Y. Sun, "Grasp planning to maximize task coverage," IJRR, vol. 34, no. 9, pp. 1195-1210, 2015.

[28] T. Mörwald, J. Prankl, A. Richtsfeld, M. Zillich, and M. Vincze, "BLORT - the blocks world robotic vision toolbox," in IEEE ICRA - 
Workshop on Best Practice in 3D Perception and Modeling for Mobile Manipulation, 2010. 